\title{
Analysis and Experimental Results of Interior DAC of SAR ADC using Cadence
}

\author{
Kalmeshwar N. Hosur ${ }^{1}$, Dr. Girish V. Attimarad ${ }^{2}$, Dr. Harish M. Kittur ${ }^{3}$ \\ ${ }^{I}$ Department of Electronics \& Communication Engg., S.D.M. College of Engineering \& Technology, Dharwad, \\ Karnataka, India, \\ ${ }^{2}$ Department of Electronics \& Communication Engg., Dayanand Sagar College of Engineering, Kumar swamy \\ Layout, Bangalore, Karnataka, India , \\ ${ }^{3}$ School of Electrical Sciences, VIT University, Vellore, Tamilnadu, India .
}

\begin{abstract}
This paper focuses on analysis and experimental results of 6-bit charge-redistribution DAC and 6-bit charge-redistribution DAC using split array configuration. These DAC configurations are designed and simulated using GPDK 180nm CMOS technology. DAC is an important component within the SAR ADC. The charge redistribution DAC in a Split capacitor configuration has a total capacitance which is $87.5 \%$ smaller compared to a conventional design. Hence DAC gives the optimized architecture. Optimized design of DAC architecture ensures the accuracy of the components, which improves the performance of SAR ADC. The matching accuracy of integrated capacitors is excellent. The simulation results of both are compared. The delay required to get the output is 793.7E-15S and 793.6E-15S when all input bits are high for charge-redistribution $D A C$ and split array DAC respectively. Dynamic range for these DACs is $35.98 \mathrm{~dB}$. The supply voltage is $1.8 \mathrm{~V}$.

Keywords: Analog-to-Digital converter, Digital- to- Analog converter, Charge redistribution, Successive Approximation, Split array.
\end{abstract}

\section{Introduction}

Successive Approximation Register Analog-to-Digital Converter (SAR ADC) compares the input voltage and output voltage of an N-bit digital-to-analog converter (DAC) with only one comparator. The output voltage of DAC is used as a reference voltage; SAR ADC can get the final conversion result through a total of $\mathrm{N}$ times comparison. SAR ADC has moderate accuracy and moderate speed. It could achieve smaller chip area and low power consumption with CMOS technology. SAR ADC is widely used in industrial control, medical instrumentation, microprocessor-assisted analog-digital conversion interfaces and other fields. SAR ADC linearity is mainly limited by the linearity of built-in DAC and the structure of DAC. The match precision of devices are the decision factors for ADC's performance. Therefore, it is the key component to ensure the performance of SAR ADC that design a DAC with considerable accuracy for the overall system [1]. In recent work on SAR ADCs a new Digital-to-Analog Converter was introduced. This new converter is based on charge redistribution and it can easily be operated at very high sampling rates. Furthermore the matching accuracy of integrated capacitors is excellent. Converters up to 12-bit or even more resolution are possible without any adjustments. DACs that are used in ADCs are already adequate if they only show the right output value when the comparator action takes place [7]. The array capacitance influences the RC settling time of the DAC and therefore limits the entire converter speed. Reduction of capacitance and the die area of the DAC can be achieved by using split capacitor technique [3]

This paper describes analysis of 6-bit charge redistribution DAC and its implementation using split array technique. Section II discusses basics of charge-redistribution DAC. Section III discusses its implementation using Cadence. Section IV contains experimental results and Section V contains conclusions.

\section{Basics Of Charge Redistribution And Split Array Dacs}

\subsection{Charge redistribution DAC}

Fig.1 shows, a charge redistribution DAC is a parallel array of binary-weighted capacitors, $2^{\mathrm{N}} \mathrm{C}$ in total. After initially being discharged, the digital signal switches each capacitor to either $V_{\text {ref }}$ or ground, causing the output voltage, $V_{\text {out }}$, to be a function of the voltage division between the capacitors $[5,6]$. 


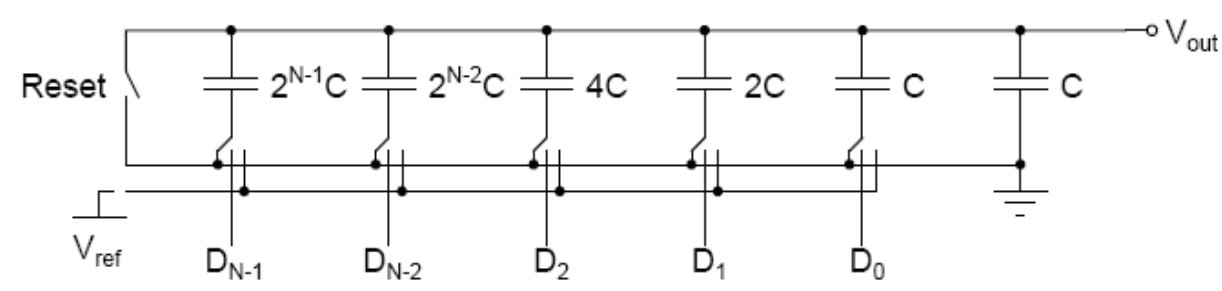

Fig. 1. A Charge-redistribution DAC

The capacitor array totals $2^{\mathrm{N}} \mathrm{C}$. Therefore, if the MSB is high and the remaining bits are low, then a voltage divider occurs between the MSB capacitor and the rest of the array. The analog output voltage, $V_{\text {out }}$, becomes:

$$
V_{\text {out }}=V_{r e f} \cdot \frac{2^{N-1} C}{\left(2^{N-1}+2^{N-2}+2^{N-3}+\ldots+4+2+1+1\right) C}=V_{r e f} \cdot \frac{2^{N-1} C}{2^{N} C}=\frac{V_{r e f}}{2}
$$

which confirms the fact that the MSB changes the output of a DAC by $1 / 2 V_{\text {ref. }}$. Fig. 2 shows the equivalent circuit under this condition.

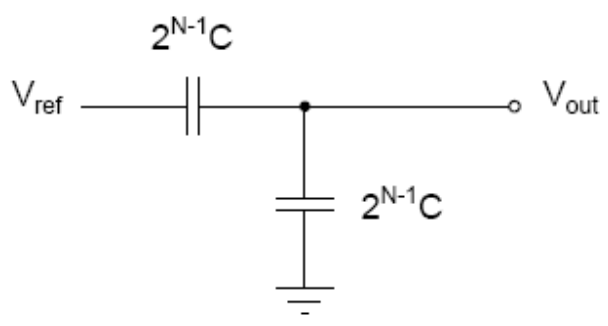

Fig. 2. Equivalent circuit with the $\mathrm{MSB}=1$, and all other bits set to zero

The ratio between $V_{\text {out }}$ and $V_{\text {ref }}$ due to each capacitor can be generalized to:

$$
V_{\text {out }}=\frac{2^{k} C}{2^{N} C} \cdot V_{r e f}=2^{k-N} \cdot V_{r e f}
$$

Where it is assumed that the $k$-th bit, $D_{k}$, is one and all other bits are zero. Superposition can then be used to find the value of $V_{\text {out }}$ for any input word by:

$$
V_{\text {out }}=\sum_{k=0}^{N-1} D^{k} 2^{k-N} . V_{\text {ref }}
$$

\subsection{Split array DAC}

The charge-redistribution architecture is very popular because of its simplicity and relative good accuracy. Although a linear capacitor is required, high resolution in the 10- to 12-bit range can be achieved. However, as the resolution increases, the size of the MSB capacitor becomes a major concern. For example if the unit capacitor, $C$, were $0.5 \mathrm{pF}$, and a 12-bit DAC were to be designed, the MSB capacitor would need to be:

$$
\mathrm{C}_{\mathrm{MSB}}=2^{\mathrm{N}-1} \cdot 0.5 \mathrm{pF}=1024 \mathrm{pF}
$$

One method of reducing the size of the capacitors is to use a split array. A 6-bit example of the array is shown in Fig. 3. This architecture is slightly different from the charge-redistribution DAC shown in Fig. 1 in that the output is taken off a different node and an additional attenuation capacitor is used to separate the array into a LSB array and a MSB array. Note that the LSB, $\mathrm{D}_{0}$, now corresponds to the leftmost switch and that the $\mathrm{MSB}, \mathrm{D}_{5}$, corresponds to the rightmost switch $[5,6]$. 


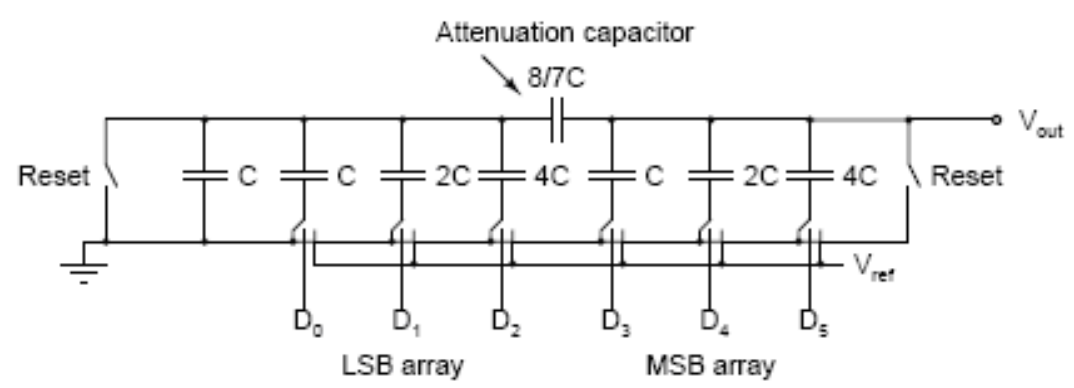

Fig. 3. A Charge-redistribution DAC using a split array

The value of the attenuation capacitor can be found by:

$\mathrm{C}_{\mathrm{att}}=$ (Sum of the LSB array capacitors / sum of the MSB array capacitors) $* \mathrm{C}$

Where the sum of the MSB array equals the sum of LSB capacitor array minus $C$. The value of the attenuation capacitor should be such that the series combination of the attenuation capacitor and the LSB array, assuming all bits are zero, equals $C$. To prove this a derivation is made, refer to formula (6). The output voltage is defined as the attenuation factor times the LSB bits plus the MSB bits times the reference voltage. The attenuation factor is a capacitive divider between the attenuation capacitor and the sum of the LSB capacitors. One can see that with some manipulation this is equal to formula (3).

$$
\begin{aligned}
& V_{\text {out }}=\left(\frac{\frac{2^{N / 2}}{2^{N / 2}-1}}{\frac{2^{N / 2}}{2^{N / 2}-1}+2^{N / 2}} \sum_{k=0}^{N / 2-1} D_{k} 2^{k-N / 2}+\sum_{k=N / 2}^{N-1} D_{k} 2^{k-N}\right) \cdot V_{r e f} \\
& =\left(\frac{1}{2^{N / 2}} \sum_{k=0}^{N / 2-1} D_{k} 2^{k-N / 2}+\sum_{k=N / 2}^{N-1} D_{k} 2^{k-1}\right) \cdot V_{r e f} \\
& =\left(\frac{2^{N / 2}}{2^{N / 2}} \cdot \sum_{k=0}^{N / 2-1} D_{k} 2^{k-N}+\sum_{k=N / 2}^{N-1} D_{k} 2^{k-N}\right) \cdot V_{r e f} \\
& =\left(\sum_{k=0}^{N / 2-1} D_{k} 2^{k-N}+\sum_{k=N / 2}^{N-1} D_{k} 2^{k-N}\right) \cdot V_{r e f} \\
& =\sum_{k=0}^{N-1} D_{k} 2^{k-N} \cdot V_{r e f}
\end{aligned}
$$

A drawback of the split array is that spreading in the attenuation capacitor affects all the capacitors after the attenuation capacitor. Therefore, care in the layout should be taken.

\section{Implementation Of Charge Redistribution And Split Array Dacs}

The 6-bit charge-redistribution DAC architecture is shown in fig. 4. The unit capacitance $\mathrm{C}=0.5 \mathrm{pF}$ is used. Total capacitance used in this DAC architecture is $32 \mathrm{pF}$. The input pulses are applied to $\mathrm{D}_{5}, \mathrm{D}_{4}, \mathrm{D}_{3}, \mathrm{D}_{2}, \mathrm{D}_{1}, \mathrm{D}_{0}$ and their specifications are mentioned in Table I .

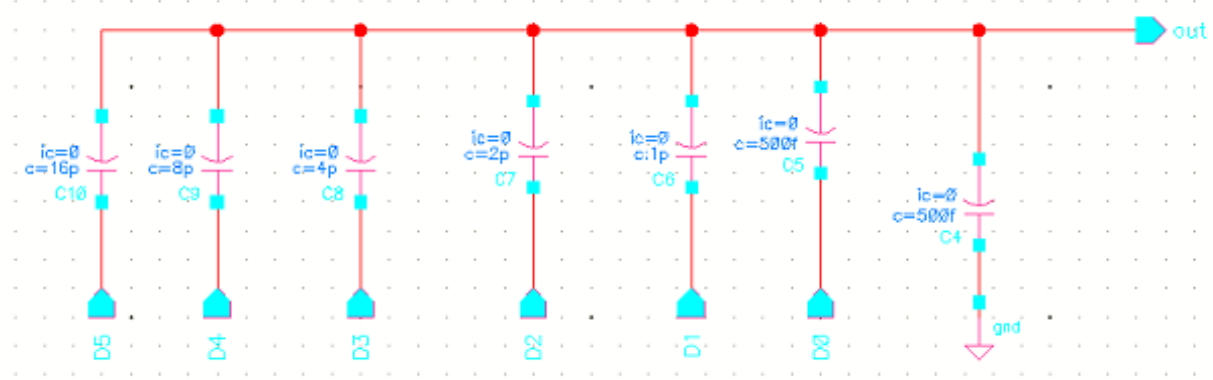

Fig. 4. 6 bit charge redistribution DAC schematic.

The 6-bit charge-redistribution DAC using a split array is shown in fig. 5. The unit capacitance $\mathrm{C}=$ $0.5 \mathrm{pF}$ is used and total capacitance of this circuit is $4 \mathrm{pF}$. The input pulses are applied to $\mathrm{D}_{5}, \mathrm{D}_{4}, \mathrm{D}_{3}, \mathrm{D}_{2}, \mathrm{D}_{1}, \mathrm{D}_{0}$ and their specifications are mentioned in Table I . The symbol view of split array DAC is shown in fig. 6. 


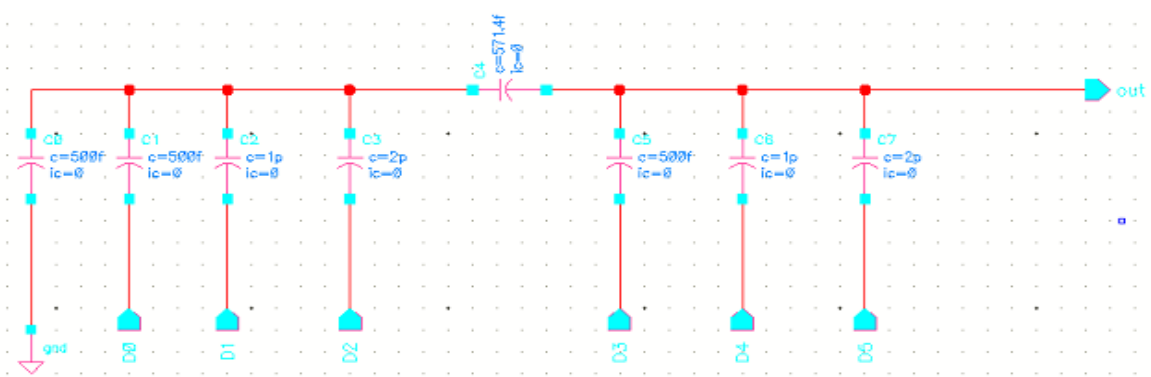

Fig. 5. A 6 Bit charge-redistribution DAC using a Split array schematic.

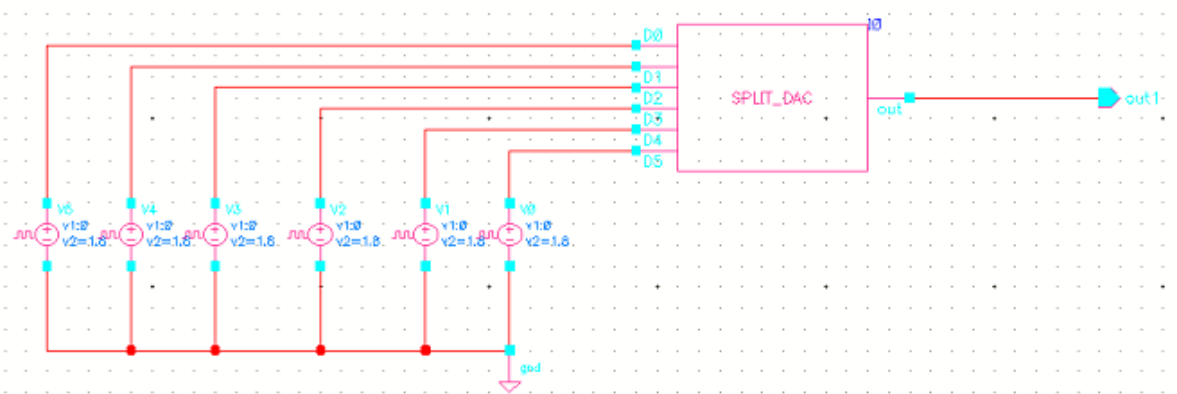

Fig. 6. A symbol view of 6 Bit charge-redistribution DAC using a split array

\section{Experimental Results}

The simulation results of 6-bit charge-redistribution DAC are shown in the fig. 7. For 6-bit chargeredistribution DAC using a split array, the results are shown in fig. 8. The Input pulses are shown in Table I for $\mathrm{D}_{5}, \mathrm{D}_{4}, \mathrm{D}_{3}, \mathrm{D}_{2}, \mathrm{D}_{1}, \mathrm{D}_{0}$ and results are shown in Table II for some input bit combinations. The delay calculated is 793.7 E-15S, and 793.6E-15S for charge-redistribution and using split array respectively, this is the time required to get output after applying inputs when all input bits are high.

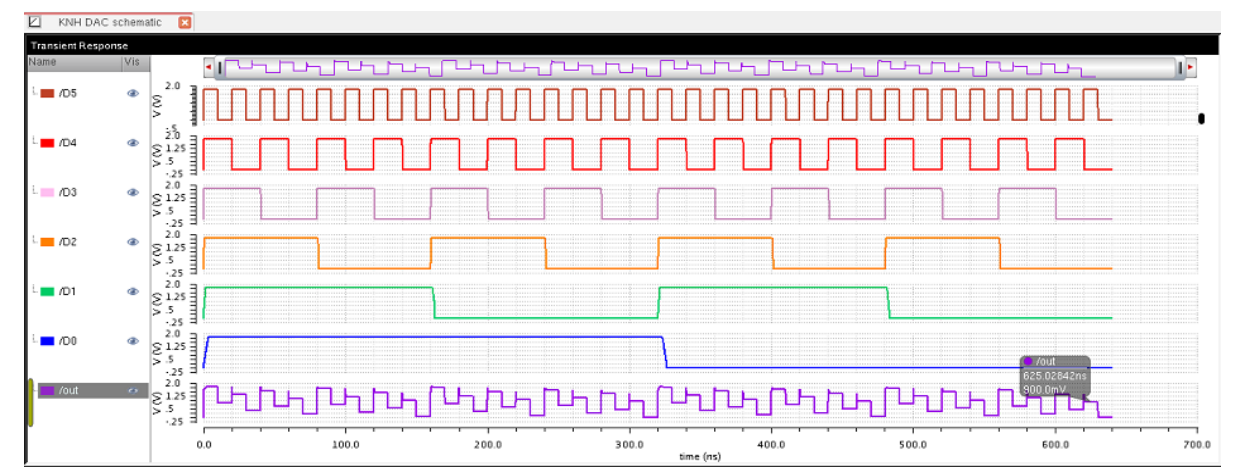

Fig. 7. Simulation Results of 6 Bit charge-redistribution DAC

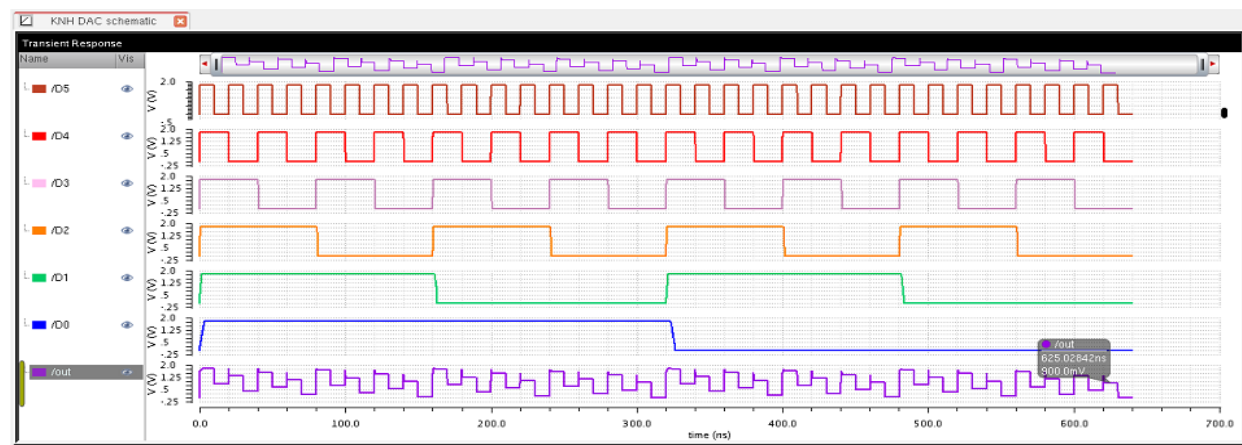

Fig. 8. Simulation results of 6 Bit charge-redistribution DAC using a Split array 


\begin{tabular}{|l|l|}
\multicolumn{2}{l}{ TABLE I: INPUT PULSES } \\
\begin{tabular}{|l|l|}
\multicolumn{1}{|c|}{ Inputs } & \multicolumn{1}{c|}{ Specifications } \\
\hline $\mathrm{D}_{5}(\mathrm{MSB})$ & $\mathrm{V} 1=0 \mathrm{v}, \mathrm{V} 2=1.8 \mathrm{~V}$, Ton $=10 \mathrm{~ns}, \mathrm{~T}=20 \mathrm{~ns}$ \\
\hline $\mathrm{D}_{4}$ & $\mathrm{~V} 1=0 \mathrm{v}, \mathrm{V} 2=1.8 \mathrm{~V}, \mathrm{~T}$ on $=20 \mathrm{~ns}, \mathrm{~T}=40 \mathrm{~ns}$ \\
\hline $\mathrm{D}_{3}$ & $\mathrm{~V} 1=0 \mathrm{v}, \mathrm{V} 2=1.8 \mathrm{~V}, \mathrm{~T}$ on $=40 \mathrm{~ns}, \mathrm{~T}=80 \mathrm{~ns}$ \\
\hline $\mathrm{D}_{2}$ & $\mathrm{~V} 1=0 \mathrm{v}, \mathrm{V} 2=1.8 \mathrm{~V}, \mathrm{~T}$ on $=80 \mathrm{~ns}, \mathrm{~T}=160 \mathrm{~ns}$ \\
\hline $\mathrm{D}_{1}$ & $\mathrm{~V} 1=0 \mathrm{v}, \mathrm{V} 2=1.8 \mathrm{~V}$, Ton $=160 \mathrm{~ns}, \mathrm{~T}=320 \mathrm{~ns}$ \\
\hline $\mathrm{D}_{0}(\mathrm{LSB})$ & $\mathrm{V} 1=0 \mathrm{v}, \mathrm{V} 2=1.8 \mathrm{~V}$, Ton $=320 \mathrm{~ns}, \mathrm{~T}=640 \mathrm{~ns}$ \\
\hline
\end{tabular}
\end{tabular}

TABLE II: ANALOG OUTPUT VOLTAGES FOR SOME INPUT BIT COMBINATIONS

\begin{tabular}{|c|c|c|}
\hline $\begin{array}{c}\text { Input bits } \\
\mathrm{D}_{5} \mathrm{D}_{4} \mathrm{D}_{3} \mathrm{D}_{2} \mathrm{D}_{1} \mathrm{D}_{0}\end{array}$ & $\begin{array}{c}\text { Output of } \\
\text { Split DAC }\end{array}$ & $\begin{array}{c}\text { Output of } \\
\text { Binary } \\
\text { weighted } \\
\text { DAC }\end{array}$ \\
\hline 000000 & $-159.33 \mathrm{nV}$ & $-17.896 \mathrm{~V}$ \\
\hline 100000 & $900.00 \mathrm{mV}$ & $900.00 \mathrm{mV}$ \\
\hline 010000 & $450.00 \mathrm{mV}$ & $450.00 \mathrm{mV}$ \\
\hline 110000 & $1.35 \mathrm{~V}$ & $1.35 \mathrm{~V}$ \\
\hline 001000 & $225.00 \mathrm{mV}$ & $225.00 \mathrm{mV}$ \\
\hline 111111 & $1.7719 \mathrm{~V}$ & $1.7719 \mathrm{~V}$ \\
\hline
\end{tabular}

\section{Conclusion}

The designed DACs are simulated using GPDK 180nm CMOS technology. The results of 6-bit chargeredistribution DAC and its implementation using split array technique are compared. The delay required to get the output for 6-bit charge-redistribution DAC is $793.7 \mathrm{E}-15 \mathrm{~S}$ and for a split array is $793.6 \mathrm{E}-15 \mathrm{~S}$. Dynamic range for these architectures is $35.98 \mathrm{~dB}$. The total capacitance required for 6-bit charge-redistribution DAC is $32 \mathrm{pF}$ and for split array is $4 \mathrm{pF}$ for a unit capacitance of $0.5 \mathrm{pF}$. The charge redistribution DAC in a Split capacitor configuration has a total capacitance which is $87.5 \%$ smaller compared to a conventional design. Hence split array DAC is optimized one.

\section{Acknowledgements}

We thank the management, Principal, Staff of S.D.M College of Engineering and Technology, Dharwad, Karnataka, India, for encouraging us for this research work.

\section{Conference Papers:}

\section{References}

[1] PEI Xiaomin and ZHANG Jun, "Design and Optimization on the Interior DAC of SAR ADC", 2011 International Conference on Information Management and Engineering (ICIME 2011), IPCSIT vol. 52 (2012) C) (2012) IACSIT Press, Singapore

[2] Dariusz, Kościelnik, Marek, Miśkowicz, "Modeling event-driven successive charge redistribution in ADC with varying rate of charge transfer", 2012 IEEE 27th convention of Electrical and Electronics Engineers in Israel.

\section{Journal Papers}

[3] Olli Kursu and Timo Rahkonen, "Charge Scaling 10-bit Successive Approximation A to D Converter with Reduced Input Capacitance", 978-1-4577-05168/11/\$26.00@2011 IEEE.

[4] Michiel van Elzakker, Member, IEEE, Ed van Tuijl, Member, IEEE, Paul Geraedts, Daniël Schinkel, Member, IEEE, Eric A. M. Klumperink, Senior Member, IEEE, and Bram Nauta, Fellow, IEEE, "A 10-bit Charge-Redistribution ADC Consuming 1.9 uW at $1 \mathrm{MS} / \mathrm{s}$ ”, IEEE JOURNAL OF SOLID-STATE CIRCUITS, VOL. 45, NO. 5, MAY 2010

\section{Books}

[5] Phillip E. Allen and Douglas R. Holberg, "CMOS Analog Circuit Design”, Second Edition, New York Oxford, Oxford University press 2004.

[6] Jacob Baker, Harry W.Li and David E.Boyce, "CMOS Circuit Design, Layout, and Simulation”, IEEE Press, Prentice Hall of Thesis India Private Limited, New Delhi, 2000.

[7] Frank B. Boschker MSc, "Design of a 12 bit 500Ms/s standalone charge redistribution Digital-to-Analog Converter" MSc. Thesis January 2008. 